\title{
Graph Matching: Fast Candidate Elimination Using Machine Learning Techniques
}

\author{
M. Lazarescu ${ }^{1,2}$, H. Bunke ${ }^{1}$, and S. Venkatesh ${ }^{2}$ \\ 1 Computer Science Department, University of Bern, Switzerland \\ 2 School of Computing, Curtin University, Perth, Australia \\ lazaresc@cs.curtin.edu.au, bunke@iam.unibe.ch, svetha@fury.cs.curtin.edu.au
}

\begin{abstract}
Graph matching is an important class of methods in pattern recognition. Typically, a graph representing an unknown pattern is matched with a database of models. If the database of model graphs is large, an additional factor in induced into the overall complexity of the matching process. Various techniques for reducing the influence of this additional factor have been described in the literature. In this paper we propose to extract simple features from a graph and use them to eliminate candidate graphs from the database. The most powerful set of features and a decision tree useful for candidate elimination are found by means of the $\mathrm{C} 4.5$ algorithm, which was originally proposed for inductive learning of classification rules. Experimental results are reported demonstrating that efficient candidate elimination can be achieved by the proposed procedure.
\end{abstract}

Key words. Structural pattern recognition, graph matching, graph isomorphism, database retrieval, database indexing, machine learning, C4.5

\section{Introduction}

In structural pattern recognition, graphs play an important role for pattern representation. Typically, objects or parts of objects are represented by means of nodes, while information about relations between different objects or parts of objects is captured through edges. Thus structural relationships can be represented in an explicit way. When graphs are used for pattern representation, the recognition problems turn into the task of graph matching. I.e., a graph extracted from an unknown input object is matched to a database of model graphs to recognize or classify the unknown input [123. Application examples of graph matching include character recognition 4,5, schematic diagram interpretation [6 7], shape analysis [8] and 3-D object recognition [9].

Structural pattern recognition by means of graph matching is attractive because graphs are a universal representation formalism. But on the other hand, graph matching is expensive from the computational complexity point of view. Matching two graphs with each other requires time and space exponential in the number of nodes involved. An additional problem arises if the database of 
model graphs is large because straightforward, sequential comparison of the unknown input with each graph in the database results in an additional factor in the complexity, which is proportional to the database's size.

Various indexing mechanisms have been proposed to reduce the complexity of graph matching in case of large databases [3.12 1011. In this paper we propose a new approach based on machine learning techniques. For the purpose of simplicity, only the problem of graph isomorphism detection is considered. The main idea of the proposed approach is to use simple features, which can be efficiently extracted from a graph, to reduce the number of possible candidates in the database. Examples of such features are the number of nodes or edges in a graph, the number of nodes or edges with a certain label, the number of edges (with a certain label $l$ ) incident to a node (with a certain label $l^{\prime}$ ), a.s.o. Obviously, a necessary condition for a graph in the database being isomorphic to the input graph is that these features have identical values in both graphs. Therefore, it can be expected that certain graphs in the database can be ruled out by a few fast tests using only these simple features. Consequently, the number of candidates that have to undergo an expensive test for isomorphism can be reduced.

A potential problem with this approach is that there may exist a large number of simple features. Hence the questions arises which of these features are most suitable to rule out as many candidates from the database as quickly as possible. To find the best feature set, we propose the application of machine learning techniques in this paper. In particular, the well-known $\mathrm{C} 4.5$ algorithm will be used in the context of this paper [13].

In the next section, a brief introduction to $\mathrm{C} 4.5$ will be provided. Then the application of $\mathrm{C} 4.5$ to reducing the number of candidate graphs in a database will be described. Experimental results will be presented in Section 4, and conclusions drawn in Section 5 .

\section{Introduction to $\mathrm{C} 4.5$}

C4.5 is a program that generates decision trees 13 . The reason for selecting C4.5 for this work is because it is one of the best known programs of this kind. $\mathrm{C} 4.5$ is based on the divide and conquer paradigm described in the following. Let $S$ be the set of training instances and let the classes be $C_{1}, C_{2}, C_{3}, \ldots, C_{n}$. There are 3 cases:

- $S$ contains one or more instances which all belong to a class $C_{j}$. The decision tree for $S$ is a leaf identifying $C_{j}$.

- $S$ is empty. The decision tree for $S$ is a leaf, but the class associated with the leaf must be determined from information other than $S$. For example, the class is chosen based on background information of the domain such as the overall majority class.

- $S$ contains instances that belong to a mixture of classes. In this case, $S$ is refined into subsets of instances that are or seem to be heading towards a single-class collection of cases. A test $T$ is chosen, based on a single attribute, 
that has one or more mutually exclusive outcomes $O_{1}, O_{2}, O_{3}, \ldots, O_{l}$ and $S$ is partitioned into subsets $S_{1}, S_{2}, S_{3}, \ldots, S_{l}$ where $S_{j}$ contains all the instances in $S$ that have outcome $O_{j}$ in the chosen set. The decision tree for $S$ now consists of a decision node identifying the test and one branch for each possible outcome. The same process is applied to each subset of the training cases so that the $j$-th branch leads to a decision tree constructed from the subset $S_{j}$ of the training instances.

In the particular application considered in this paper, each graph in the database corresponds to exactly one class $C_{i}$, and $S=C_{1} \cup C_{2} \cup \ldots \cup C_{n}$ is the set of prototype graphs in the database.

Partitioning at each level in the decision tree is achieved by finding the attribute that maximizes the information gained from choosing that attribute. C4.5 uses a normalized gain criterion to select which attribute should be used. The definition of the gain ratio used by $\mathrm{C} 4.5$ is defined below:

$$
\begin{gathered}
\text { gain-ratio }(X)=\frac{\operatorname{gain}(X)}{\operatorname{split-info}(X)}, \text { where } \\
\text { split-info }(X)=\sum_{i=1}^{l} \frac{\left|T_{i}\right|}{|T|} \times \log _{2}\left(\frac{\left|T_{i}\right|}{|T|}\right), \\
\operatorname{gain}(X)=\operatorname{info}(T)-\operatorname{info}_{x}(T), \\
\operatorname{info}(T)=\sum_{j=1}^{l} \frac{\operatorname{freq}_{j}\left(C_{j}, T\right)}{|T|} \times \log _{2}\left(\frac{\operatorname{freq}_{j}\left(C_{j}, T\right)}{|T|}\right), \\
\left.\operatorname{info}_{x}(T)=\sum_{i=1}^{n} \frac{\left|T_{i}\right|}{|T|} \times \operatorname{info}_{i}\right)
\end{gathered}
$$

and $T$ is the set of instances, split into $l$ outcomes based on the test $X$ with $C_{j}$ being some random class. The frequency ratio - freq $\left(C_{j}, T\right) /|T|-$ represents the probability that given the case in which a training instance is taken at random from the training set $T$, that training instance belongs to class $C_{j}$.

Attribute values can be discrete or real-valued and $\mathrm{C} 4.5$ can handle cases involving unknown or imprecise values. C4.5 always attempts to develop the simplest decision tree that is representative of the data and when necessary, it can apply sophisticated pruning techniques to reduce the size of the tree. For further detail see [13]. 


\section{Graph Candidate Elimination Using C4.5}

Given an input graph $g$ and a database with $n$ model graphs $g_{1}, g_{2}, \ldots, g_{n}$ where each $g_{i}$ represents a pattern class $C_{i}$, we consider the problem of finding a graph $g_{j}$ in the database that is isomorphic to $g$. Formally, $g$ and $g_{j}$ are isomorphic if there exists a bijective mapping $f$ from the nodes of $g$ to the nodes of $g_{j}$ such that the structure of the edges and all node and edge labels are preserved under $f$. As described in the introduction, we are interested in a procedure that efficiently reduces the number of potential candidate graphs in the database.

Our method for graph candidate elimination has three stages. In the first stage we extract the features from the graphs. There are many features that can be potentially used, ranging from simple features such as the number of vertices or edges per graph to complex ones such as chains of vertices and edges with given labels. For our research we have selected the three types of features listed below:

- number of vertices with a given label (feature-type 1).

- number of incoming edges per vertex (feature-type 2).

- number of outgoing edges per vertex (feature-type 3).

The reason for selecting these features is that they are easy and computationally inexpensive to extract. For a graph with $m$ vertices, the extraction takes only $O\left(\mathrm{~m}^{2}\right)$ time and space. On the other hand, as will be shown later, these features are very efficient in ruling out potential candidate graphs. For example, if the input graph has three vertices of a given label $A$, then the search for isomorphic graphs can be restricted only to those graphs in the database that have exactly three vertices with the label $A$. Once the features have been extracted, they are passed on to C4.5.

The second stage involves the use of $\mathrm{C} 4.5$ to build the decision tree based on any combination of the three types of features mentioned above.

The third stage is to determine which graphs can possibly match a given input graph. Obviously, a necessary condition for input graph $g$ being isomorphic to model graph $g_{j}$ is that all features extracted from $g$ have values identical to the corresponding features extracted from $g_{j}$. The processing is as follows. First, we extract from the input graph the same features that were extracted from the graphs in the database. Then we use the values of the features extracted from the input graph to traverse the decision tree. There are only two possible outcomes. The first outcome is that we reach a leaf node. In this case the graphs associated with the leaf node are possible matches to the input graph. Each of these graphs is tested against the input for graph isomorphism using a conventional algorithm, for example the one reported in [14. The second outcome is that we do not reach a leaf node. In this case there are no graphs in the database that can be isomorphic to the input graph.

The cost to extract any of the features mentioned above from a graph with $m$ vertices is $O\left(m^{2}\right)$. The cost to traverse a decision tree of depth $k$ is $O(k)$ while the cost to match a given input graph against a set of $c$ candidate graphs 
is $O\left(c \cdot m^{m}\right)$. Hence, for a database consisting of $n$ graphs of size $m$ each, the cost to find a match for an input graph is $\left[O\left(c \cdot m^{m}\right)+O\left(m^{2}\right)+O(k)\right]$ given that the decision tree has $k$ levels and the maximum number of graphs associated with a leaf in the decision tree is $c$. The graphs associated with a leaf node in the decision tree will be called a cluster in the following. We observe that the expression

$$
O\left(c \cdot m^{m}\right)+O\left(m^{2}\right)+O(k)
$$

has to be compared to

$$
O\left(n \cdot m^{m}\right)
$$

which is the computational complexity of the straightforward approach, where we match the input graph to each element in the database. Because $c<n$ or $c<<n$, a significant speedup over the straightforward approach can be expected.

\section{Experimental Results}

The two parameters that are related to decision trees and have a significant impact on the overall efficiency of the method are the cluster size $c$ and the depth of the decision tree $k$. We have investigated the two parameters by conducting a number of experiments on a database of randomly generated graphs. The parameters used in the graph generation process were the number of vertices, the number of vertices, the number of edges per graph and the number of graphs in the database.

As the influence of edge labels can be expected similar to the influence of node labels, no edge labels were used. The experiments were conducted as follows. First a database of random graphs was generated. Then the features were extracted from the graphs and passed on to $\mathrm{C} 4.5$ which constructed a decision tree to classify the graphs in the database. In the final step of the experiment, the decision tree was analyzed and the average cluster and largest cluster size recorded along with the depth of the decision tree.

In the first set of experiments we focused on the first type of feature extracted from the graphs, i.e. the frequency of the various vertex labels in the graphs. Therefore the decision tree classifying the graphs in the database was built using information from only this type of feature. To extract the feature we used a simple histogram that recorded the occurrence of each given vertex label. We started with only 5 vertex labels and then gradually increased the number of labels to 100 . We also varied the number of vertices per graph from 5 to 50 . At the same time, the number of edges was varied from 8 to 480. The number of graphs in the database was 1000 in this experiment. All graphs in the database had the same number of nodes and edges. Figure 1 shows the average cluster size. The results indicate that as the number of vertices increases, the average cluster size decreases. In the majority of the experiments, the average cluster size converged to the value of 2 which means that in general only 2 graphs out 

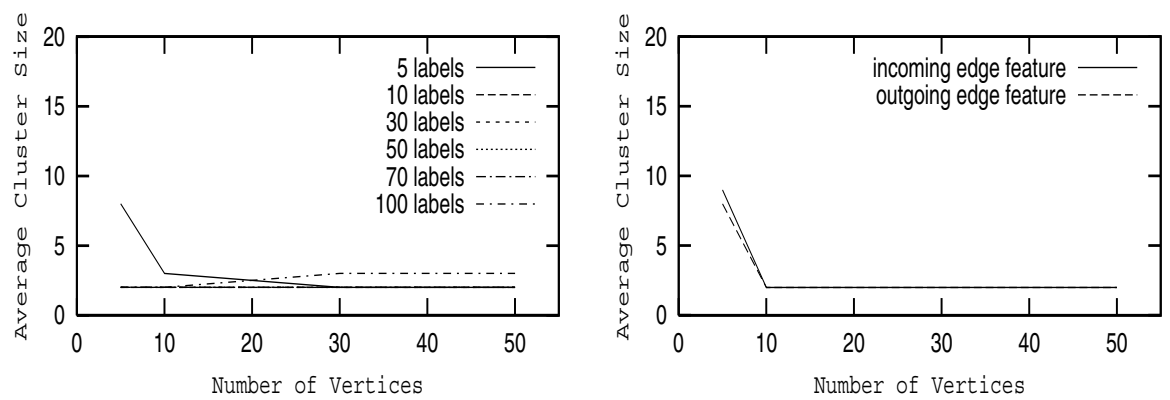

Fig. 1. (left) Using the vertex feature (1000 graphs).

Fig. 2. (right) Using the incoming/outgoing edge features (1000 graphs).

of a 1000 will be needed to conduct a test for graph isomorphism. The fact that the size of the cluster decreases as the number of vertices or labels increases was expected since the greater the number of vertices/labels per graph is, the more easily identifiable a graph becomes. Hence C4.5 is able to develop better classification trees with a smaller cluster size.

The second set of experiments were done using the frequency of edges incident per vertex. Both incoming and outgoing edges were considered in separate experiments. Again, the number of vertices was increased from 5 to 50 . At the same time, the number of edges was varied from 8 to 480. The database's size was 1000. No label information was used in these experiments. Figure 2 shows the average cluster size for both the case in which the incoming and outgoing edges were used. The results are similar to those obtained from the case in which feature-type 1 was used, in the sense that the greater the number of vertices, the smaller the cluster size.

In the third set of experiments, we used pairs of features to build the decision tree classifying the graphs in the database. Three pairs of features were used: (feature-type 1,feature-type 2), (feature-type 1,feature-type 3) and (feature-type 2,feature-type 3). We used the same parameters as for feature-type 1: 1000 graphs per database, 5-50 nodes per graph with all graphs in the database having the same size, and 5-100 vertex labels. The results obtained from combining feature-type 1 and feature-type 2 are shown in Fig. 3. The average cluster size has value of 2 for all cases except when the graphs were of size 5 (the average cluster for this case was 3). These results are better than those in the case in which any of the three single features were used for classification. The reason for this is that by using two features more information about the graphs becomes available, and $\mathrm{C} 4.5$ was able to classify the graphs with more accuracy. The results for the last 2 pairs of features (feature-type 1,feature-type 3 ) and (featuretype 2,feature-type 3) were very similar. In both cases the average cluster size was 2 and in both cases the results are better than in the case where only a single feature was used to classify the graphs. In the fourth set of experiments, we combined all three types of features. The results obtained are shown in Fig. 4 , 

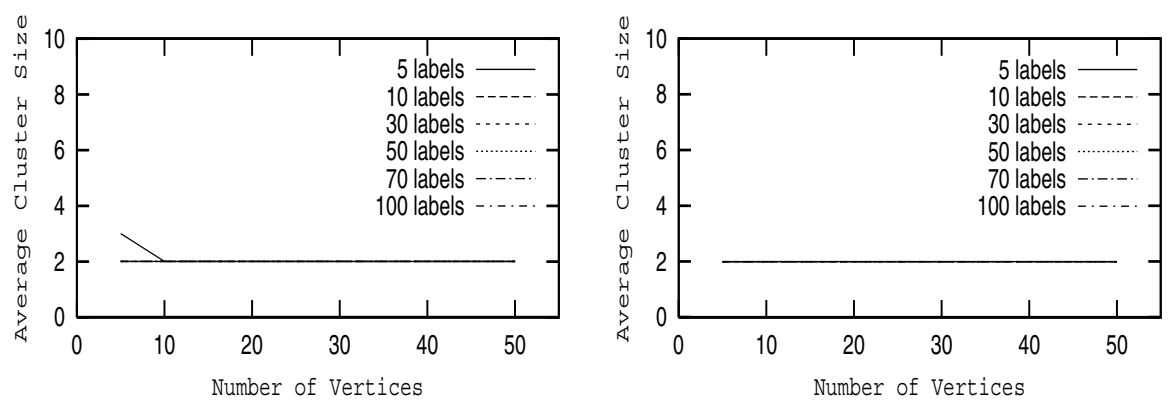

Fig. 3. (left) Using the feature combination 1-2 (1000 graphs).

Fig. 4. (right) Using the feature combination 1-2-3 (1000 graphs).

Note that this time the average cluster size had a constant value of 2 regardless of the size of the graphs. Therefore the results are better than those obtained for the cases in which single features or pairs of features were used by $\mathrm{C} 4.5$ to build the decision tree. This result matches our expectation since more information is available and this leads to more accurate decision trees being built.

For each of the experiments described previously, we not only recorded the cluster size but also the depth of the decision tree. When feature-type 1 was used, the depth of the decision tree varied as shown in Fig. 囵. In this case, when the number of vertices is small ( 5 vertices per graph) and the number of vertex labels is high (100 labels), the depth of the decision tree is large (over 40). As the number of vertices increase and the number of vertex labels decrease, the depth of the decision tree decreases until it finally converges to an average depth of 11. When feature-type 2 and feature-type 3 were used, the depth of the decision trees was more constant and not influenced by the number of vertices in the graphs (see Fig. 6). The average decision tree depth is 11, which is the same as in the case of feature-type 1.
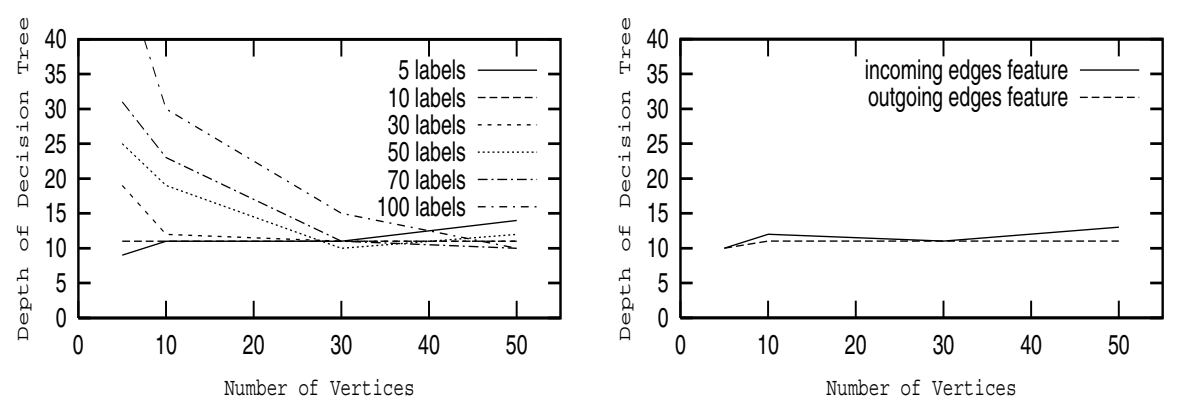

Fig. 5. (left) Using the vertex feature (1000 graphs).

Fig. 6. (right) Using the incoming/outgoing edge features (1000 graphs). 

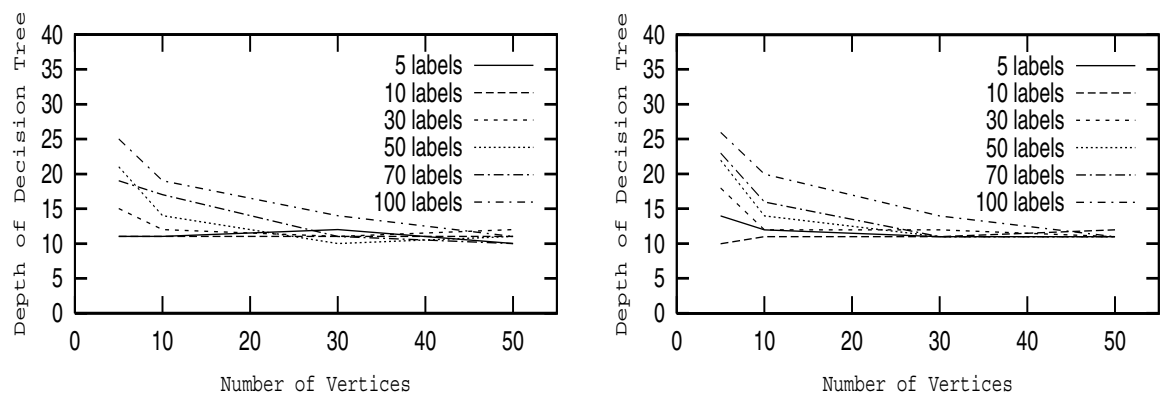

Fig. 7. (left) Using the feature combination 1-2 (1000 graphs).

Fig. 8. (right) Using the feature combination 1-3 (1000 graphs).

The results obtained from using pairs of features are shown in Figs. 7,8 and 9. In all three cases there is a substantial improvement when compared with results obtained using feature-type 1. The depth of the tree converges faster to an average value of 11 and in the worst case (small number of vertices, high number of labels) the decision tree depth is only 25 . Therefore combining features together not only helps in reducing the average cluster size but also helps reduce the depth of the tree. The results also show that there were no substantial differences between the three pairs of features.

More improvement can be obtained by using more types of features. This is indicated by the results produced when using feature-types 1-3 together (see Fig. (10). In this case the depth of the tree converges faster to an average depth of 11. Also when more features are used, the depth of the tree in the worst case scenario (small number of vertices, high number of labels) is less than 25. This is a reduction of almost 50 when one considers the case of feature-type 1 .

To give some concrete hints about the computation time that can be saved by the method proposed in this paper, we measured the quantities in eqs. (II)
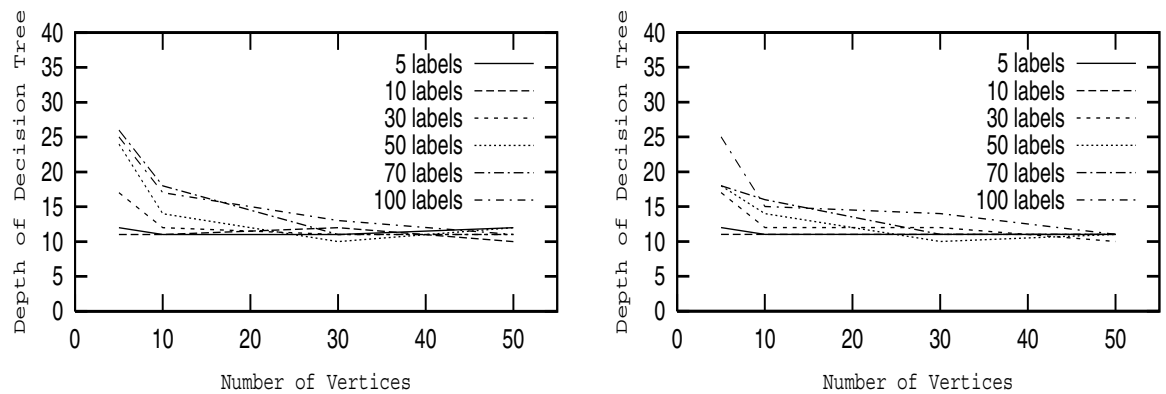

Fig. 9. (left) Using the feature combination 2-3 (1000 graphs).

Fig. 10. (right) Using the feature combination 1-2-3 (1000 graphs). 
and (2) on a set of graphs with 20 vertices each. On a standard workstation, it takes about 20s to match an input graph with a database of 1000 graphs using an implementation of Ullman's algorithm [14. Traversing a decision tree of depth 11 takes $0.3 \mathrm{~s}$, and feature extraction takes $0.08 \mathrm{~s}$. Thus if we neglect the time needed off-line for decision tree construction, only $0.4 \mathrm{~s}$ are needed by our method as compared to 20s for a full search. Because of the exponential complexity of graph matching, a higher speedup can be expected for larger graphs.

\section{Conclusions}

In this paper we have presented a novel approach to graph matching that involves the use of decision trees. The method offers the advantages that it significantly reduces the number of candidate graphs in the database and it requires features that are easy to extract. It involves building a decision tree that classifies the graphs along selected features and, in order to find a match for a given graph one simply needs to go down the decision tree. In the best case scenario the leaf node (cluster) of the decision tree will contain just one graph while in the worst case it will contain several graphs. The complexity of the method is determined by 3 parameters: the size of the cluster associated with the leaf node in the decision tree, the size of the graph and the depth of the decision tree.

We have conducted numerous experiments to investigate the average cluster size at the leaf node and the depth of the decision tree. We used a database size of 1000 graphs. The graphs in the database had between 5 and 50 vertices and the set of vertex labels varied between 5 and 100 labels. The results indicate that the average cluster size is directly affected by the number of vertices and vertex labels in the graphs. The higher the number of vertices per graph, the smaller is the average cluster size. Also the higher the number of vertex labels, the smaller is the average cluster size. Another way of keeping the size of the clusters minimal is to use combinations of features. Combinations of two and three features generally produce increasingly better results.

The method proposed in this paper can lead to substantial savings in computation time when matching a graph to a database of models. Both feature extraction and decision tree traversal are very fast. At the same time they are suitable for eliminating a large portion of candidates from the database, leaving only a few graphs that have to undergo expensive isomorphism test.

The method presented in this paper is suitable to search for graph isomorphisms. Future work will investigate if this method can be applied to the subgraph isomorphism and the approximate graph matching problems. Future work could also involve the use of more complex features for classification. The features used for the work described in this paper are simple and easy to extract. However, there are other types of features such as vertex-edge chains that could be used to build the decision trees. Such features are more complex and harder to extract from graphs, but they offer the benefit that they provide a more unique description of each graph in the database. 


\section{Acknowledgment}

The authors want to thank Dr. X. Jiang of the University of Bern for his comments and for providing the implementation of Ullman's algorithm.

\section{References}

1. L.G. Shapiro and R.M. Haralick. Structural descriptions and inexact matching. IEEE Trans. Pattern Analysis and Machine Intelligence, volume 3, pages 504-519, 1981.

2. A. Sanfeliu and K.S. Fu. A distance measure between attributed relational graphs for pattern recognition. In IEEE Trans. Systems, Man, and Cybernetics, volume 13, pages 353-363, 1983.

3. B. Messmer and H. Bunke. A new algorithm for error-tolerant subgraph isomorphism detection. In IEEE Trans. Pattern Analysis and Machine Intelligence, volume 20, pages 493-505, 1998.

4. S.W. Lu, Y. Ren, and C.Y. Suen. Hierarchical attributed graph represenation and recognition of handwritten Chinese characters. In Pattern Recognition, volume 24, pages 617-632, 1991.

5. J. Rocha and T. Pavlidis. A shape analysis model with applications to a character recognition system. In IEEE Trans. Pattern Analysis and Machine Intelligence, volume 16, pages 393-404, 1994.

6. S.W. Lee, J.H. Kim, and F.C.A Groen. Translation-, rotation-, and scale-invariant recognition of hand-drawn symbols in schematic diagrams. In Int'l J. Pattern Recognition and Artificial Intelligence, volume 4, no. 1, pages 1-15, 1990.

7. H. Bunke, B. Messmer. Clustering and error-correcting matching of graphs for learning and recognition of symbols in engineering drawings. J. Hull and S. Taylor (eds.): Document Analysis Systems II, pages 102-117. World Scientific, 1998.

8. A. Pearce, T. Caelli, and W.F. Bischof. Rulegraphs for graph matching in pattern recognition. In Pattern Recognition, volume 27, no. 9, pages 1231-1246, 1994.

9. E.K. Wong. Model matching in robot vision by subgraph isomorphism. In Pattern Recognition, volume 25, no. 3, pages 287-304, 1992.

10. L.G. Shapiro and R.M. Haralick. Organization of relational models for scene analysis. In IEEE Trans. Pattern Analysis and Machine Intelligence, volume 3, pages 595-602, 1982.

11. K. Sengupta and K.L. Boyer. Organizing large structural modelbases. In IEEE Trans. Pattern Analysis and Machine Intelligence, volume 17, 1995.

12. B. Messmer and H. Bunke. A decision tree approach to graph and subgraph isomorphism. In Pattern Recognition, volume 32, pages 1979-1998, 1999.

13. J. R. Quinlan. C4.5 : Programs for machine learning. Morgan Kaufmann Publishers, 1993.

14. J.R. Ullman. An algorithm for subgraph isomorphism. In JACM, volume 23(1), pages 31-42, 1976. 